\title{
Los modelos de entrenadores y educadores en el escenario del deporte escolar
}

The coach and teacher models in the school sports scenery

\author{
Antonio Fraile Aranda*
}

\section{Resumen}

$\begin{array}{ll}\text { Palabras clave: } & \text { Perfiles de los entrenadores y educadores en el deporte escolar, las tradiciones en la formación de los técnicos deportivos, deporte } \\ & \text { escolar, la formación de los técnicos deportivos escolares. }\end{array}$

Entre las principales actividades para la socialización de los escolares están las prácticas deportivas, gracias a las cuales los entra nadores y educadores pueden fomentar una serie de capacidades vinculadas con los ámbitos cognitivos, físicos, afectivos, sociales y emocionales. Ya que, principalmente a través de la actividad física y deportiva los escolares pueden lograr un mejor desarroll
integral.

Considerando que la actuación pedagógica del educador deportivo es decisiva para alcanzar esa actividad deportiva, su pert? educativo vendrá determinado tanto por sus creencias y experiencias previas como jugador, como por el tipo de conocimientos adquiridos en su formación inicial, que condicionarán la forma de concebir su actuación práctica.

En el ámbito de la formación de los educadores deportivos podemos determinar varias perspectivas que proceden del ámbita de la formación de los educadores en general, cada una de ellas con un componente ideológico, tanto en el discurso teórico, com en su posterior desarrollo práctico. Dicha formación viene determinada por un conjunto de tradiciones o formas de enseñar, pot ejemplo el modelo tradicional empírico analítico, el modelo simbólico interpretativo y el modelo crítico. Éstos a su vez nos condu. cen hacia unos determinados perfiles que se vienen observando en los entrenadores y educadores deportivos, a saber: el autoritario el tradicional, el colaborativo, el permisivo, el creativo y el crítico. Abstract

Key Words:

Sport practices are among the most important activities for a school student's socialization. Thanks to them, coaches and teachery are able to foster a number of skills associated to the cognitive, physical, affective, social and emotional dimensions. It is mainly through physical and sport activities that school students may develop integrally.

Given that the pedagogical role of the sports educators is crucial to reach sport activity, their professional profile will be determined both by their own background as players and by the body of knowledge acquired during their initial training, which will shape their approach in practice.

Some perspectives based upon educators' preparation in general can be identified in the school sports educators' professional training, each one with an underlying ideology in both theoretical and practical aspects. This professional training has been shaped by a set of teaching traditions and styles, namely: the conventional empirical-analytical model, the symbolic-interpretive model, and the critical model. All of them lead to certain features of professional profile observed in coaches and sports educators alike, such as the authoritarian, traditional, collaborative, permissive, creative, and critical approaches.

Coach and educator's profile in school sports, traditions in the training of sport technicians, school sports, school sports educators'
training.

Fecha de recepción: 30 de agosto de 2006

Fecha de aceptación: 22 de noviembre 2006

* Doctor en Ciencias de la Educación y Licenciado en Educación Física. Catedrático de la Facultad de Educación de la Universidad
de Valladolid, España. 
La formación de los entrenadores y los educadores de iniciación deportiva debe seguir un proceso similar al del resto de enseñantes; debe atender la adquisición de una serie de competencias relacionadas con las tareas de planificar y programar, diseñar actividades, establecer un sistema de comunicación e interacción con los escolares, seleccionar los recursos, evaluar, etcétera.

Para Fraile (2005) la actuación didáctica del entrenador y el educador deportivos viene condicionada tanto por sus experiencias previas como jugador, como por el modelo transmitido por sus formadores en sus etapas como aprendiz. Por ello consideramos que la propuesta de formación hegemónica que actualmente viene empleándose en las escuelas de formación, de la mayor parte de las federaciones deportivas, tiene bastantes similitudes con el enfoque práctico-artesanal que se relaciona con los modelos de enseñanza más tradicionales.

No obstante, podemos llegar a definir y desarrollar varias perspectivas, cada una de ellas con un componente ideológico, tanto en el discurso teórico como en su desarrollo práctico posterior, gracias a las cuales conocer e identificar las diversas visiones que los entrenadores y los educadores deportivos ponen en práctica durante su actividad como enseñantes deportivos.

El perfil que adoptarán los entrenadores y los educadores deportivos en su práctica vendrá definido y determinado por unas características similares a los propios educadores que se mueven en el escenario escolar, ya que sus formas de actuar son similares a las prácticas docentes de la mayoría de los enseñantes. Por ello comenzaremos por revisar esos modelos docentes en que se clasifican las diversas formas de enseñar en el aula.

Concretamente, haremos mención a las diferentes tradiciones que conforman los procesos de formación del profesorado y que al poder transferirse al escenario deportivo pueden ayudarnos a establecer las diferencias entre los educadores y los entrenadores deportivos. Cada una de esas tradiciones serán objeto de una revisión ontológica, epistemológica y metodológica expresando sus finalidades, su origen y su evolución histórica; definiendo los conceptos más relevantes; señalando su presencia en el currículo docente, etc., así como las posibles críticas que se hace a cada una de ellas.

\section{Tradiciones en la formación del educador}

\section{La tradición empírico-analítica}

Esta tradición, que se apoya en la corriente filosófica del positivismo, genera una separación entre la teoría y la práctica, con la finalidad de buscar la verdad objetiva, en la cual los sujetos adoptan una actitud de neutralidad científica, a partir de una concepción estrictamente funcional y jerarquizada de la gestión educacional, y defiende el resultado y la eficacia por encima del proceso.

Históricamente el positivismo tiene su origen en los trabajos de los empiristas ingleses en el siglo XVIII, a través de la obra de August Comte (1798-1857), y continuada por psicólogos conductistas, como B. F. Skinner, que inciden en los programas y los métodos de instrucción escolar. Desde esta tradición se han venido estudiando las realidades sociales según el modelo establecido por la filosofía positivista, empleada en las ciencias naturales y que se define como una corriente filosófica que:

- Se opone al conocimiento metafísico y teológico.

- Rechaza todo lo que no se aprenda por medios no sensoriales, y es sólo válido el aprendizaje experimental como fuente de todo conocimiento.

- Se define como neutra y objetiva, donde la verdad es ajena al pensamiento del sujeto.

- Considera que el mundo social existe como una realidad empírica externa e independiente de la visión que tenemos de las cosas.

- La verdad se obtiene a partir de procedimientos técnicos validados.

- Sus postulados principales se producen a partir de hipótesis, que tienen como finalidad producir leyes universales y generalizables.

- Se apoya en el método hipotético-deductivo al establecer hipótesis, cuya valoración se efectúa comparando sus consecuencias deductivas con los resultados de lo observado y experimentado (Guba, 1985; Cook y Reichardt, 1986; Pérez Gómez, 1988; Sparkes, 1992, entre otros).

Para esta tradición, donde la realidad debe ser externa y tangible, el saber debe fundarse en el método científico, el currículo de carácter técnico evolucionará gracias a la experimentación, sometiendo la actividad docente a reglas técnicas que resuelvan los problemas diarios. La ausencia de relación entre teoría y práctica dificulta una mejor comprensión de la actividad educativa, y genera una división entre el trabajo teórico-intelectual del experto y el trabajo aplicativo del docente. La evaluación aparece como un medio de control y selección social. Así, la teoría educativa se convierte en una ciencia aplicada que se desarrolla a través de programas que llegan a su máximo grado de 
concreción por parte del educador y donde el jugador se limita a recibir y reproducir.

Tradicionalmente, desde esta perspectiva se han establecido las directrices de la formación del entrenador deportivo, según las cuales la actuación docente sigue un proceso lineal y descendente hacia el alumnado, actuando como meros transmisores del conocimiento de lo que prescribe la administración educativa y de los materiales que produce el mercado editorial, lo que genera una división entre la teoría del experto y la práctica del docente.

La actuación docente del educador, amparada por esta corriente positivista, presta atención especial a unos objetivos y contenidos que poseen una visión utilitarista del rendimiento motor (que se expresan en un lenguaje básicamente técnico y sin referentes contextuales), al control de la clase y la eficacia del proceso educativo, a un sistema de evaluación basado en pruebas cuantificadas (como son los tests físicos) y que olvida los aspectos actitudinales de la actividad física.

La investigación dominante en el deporte atiende a los aspectos físico-biológicos, a través de trabajos de fisiología, biomecánica, anatomía; por tanto, emplea procesos similares al desarrollado en las ciencias naturales, en las cuales predomina lo observable, cuantificable y medible. Este modelo está determinado por el método científico dominante en esta perspectiva, y las biociencias del deporte de competición son sus referentes principales. Igualmente, desde este modelo se controla la eficacia docente, basándose en el "proceso-producto" como enfoque tecnológico de la educación, conducente a buscar leyes universales.

Los programas de formación permanente de estos educadores reproducen prácticas adoptadas por los profesores expertos y no surgidas desde la propia investigación docente. Desde esta perspectiva, éstos actúan en la práctica resolviendo en forma rutinaria las múltiples y diversas situaciones del aula y aplicando reglas, teorías y procedimientos presentados como recetarios y que son defendidos por los sistemas de formación tecnológicos.

Desde una visión crítica a esta tradición, Habermas (1987) considera el saber positivista como base de los intereses prácticos de la acción instrumental, ya que se diseñan reglas para manipular la conducta del ser humano, con el objetivo de lograr los fines deseados, donde el valor instrumental puede convertirse en un medio para ejercitar el poder socioeducativo. Igualmente, se considera que el currículo técnico no sólo está limitado por la cultura positivista al seleccionar los contenidos, sino que también determina su metodología (Giroux, 1990).

\section{La tradición simbólico-interpretativa}

Esta tradición considera que la realidad es interna, idealista y relativa, y se desarrolla gracias a la fenomenología. Es necesaria la relación entre la teoría y la práctica a fin de dar al conocimiento un significado subjetivo. A comienzos del siglo $\mathrm{XX}$, a partir de estudios antropológicos de sociología urbana, esta tradición adquiere un peso importante dentro de las ciencias simbólicas. y emplea la observación participativa y las entrevistas. Después de la Segunda Guerra Mundial, y gracias a etnógrafos de la Universidad de Stanford y del Teachers College de Columbia (Estados Unidos), llega al ámbito de la investigación educativa, y alcanza su reconocimiento en Inglaterra a partir de la década de 1960 (Stenhouse, 1987; Elliott, 1990, entre otros).

Desde esta perspectiva se tiende a sustituir las nociones científicas de explicación, predicción y control, por las nociones interpretativas de comprensión, significación y acción. Se basa en un proceso de fenomenología social que surge como una ciencia que trata de descubrir las estructuras esenciales de la conciencia y para ello estudia los fenómenos como son experimentados, vividos y percibidos por el ser humano en su realidad, que a su vez depende del modo en que es vivida y percibida por él, considerando que cada situación posee un carácter interno, propio y personal.

Para esta tradición, la programación tiene un carácter práctico, evoluciona gracias a la interpretación que el educador hace de los problemas del aula, siguiendo un proceso abierto y flexible. Se produce una clara relación entre teoría y práctica, favorecida por la participación deliberativa del profesor, en la cual los jugadores, gracias a un interés comprensivo, construyen sus propios aprendizajes. La evaluación es iluminativa e interpretativa a fin de que el profesor realice un seguimiento procesal de su actividad docente. La teoría educativa se refuerza por una investigación ecológica en el aula que favorece los procesos de comunicación entre el profesor y el jugador.

Debemos formar a los educadores deportivos como investigadores e interpretadores de lo que sucede en la práctica, a fin de poder diseñar y evaluar mejor su propia programación deportiva, considerando que esa labor hace parte de su formación profesional.

Esta tradición, como alternativa al modelo positivista, ayuda al educador deportivo a reflexionar sobre lo que sucede en la práctica, construyendo nuevas formas de acción y nuevos modos de afrontar los problemas. Los objetivos y los contenidos como facilitadores de una visión integral del sujeto atienden a los aspectos: cogni- 
tivos, procedimentales y actitudinales, considerando la importancia del contexto escolar como punto de arranque del programa. La comunicación entre el educador y los jugadores es una de las claves que posibilitan el proceso educativo, estableciendo acuerdos en cuanto a los elementos básicos del programa. A través del desarrollo profesional se supera el conocimiento rutinario y reglado generado por la racionalidad técnica; el educador debe estar formado para observar, analizar, reflexionar y actuar sobre la práctica (Tinning, 1992).

En los planes de estudio de formación de los entrenadores deportivos deben estar conectadas la formación teórica y la práctica. La investigación en el deporte tendrá, en este caso, un carácter ecológico y naturalístico, y debe analizar las formas de diseñar los objetivos y los procesos de interacción educador-jugadores, organizar contenidos, emplear estrategias didácticas, utilizar los recursos, y estudiar los criterios en la evaluación a través de instrumentos etnográficos-cualitativos que permitan comprender la organización social y el comportamiento de los jugadores, y determinar una visión fenomenológica integral del sujeto.

Esta tradición también ha sido objeto de críticas, debido a su grado de aplicabilidad, es decir, la incapacidad de generar verdades generalizables a situaciones universales y establecer normas o leyes en función de la verificación de hipótesis; al fomento de los juicios de valor, como proceso de interpretación de algunos acontecimientos, ya que no garantiza un criterio suficiente de verdad; y, por último, al investigador, por que adopta una postura desinteresada, que rechaza cualquier interés en valorar criticamente las realidades educativas que se analizan sin intención de cambiarlas (Carr y Kemmis, 1988).

\section{La tradición crítica}

La tradición crítica se fundamenta en la ideología de lo subjetivo, según la cual la teoría se basa en la acción práctica. El miedo a que el positivismo transformara la ciencia en una técnica de la acción social determina que un grupo de investigadores, miembros de la Escuela de Francfort (Erich Fromm, Herbert Marcuse, Theodor Adorno, Jürgen Habermas, entre otros) estudien el impacto de la ciencia en el pensamiento del siglo $\mathrm{XX}$. Éstos, a su vez, se encargan de elaborar propuestas para poder emancipar a las personas del pensamiento positivista, y permitirles no sólo redefinir la ciencia y superar sus paradojas, sino también ayudarles a constituir un proceso de transformación y reconstrucción social.

lúdica pedaģógica, volumen 2, número 11, pp. 108-115, 2006.
Los seguidores de esta tradición consideran que el conocimiento científico se hace cada vez más provisional; así, los procesos de investigación, análisis y obtención de información crítica cobran mayor importancia para los docentes y las escuelas en la sociedad posmoderna. La certeza científica, basada en principios probados de aplicabilidad generalizada, debe ser remplazada y los docentes deben extraer información de la discusión entre conocimiento teórico y práctico. Así mismo, sus trabajos deben ayudar a indagar sobre la vida de los individuos, explorar la naturaleza y los límites del poder, la autoridad, la libertad, y buscar una mayor autonomía del individuo. Su objetivo no es tanto desarrollar nuevos programas, ni resolver problemas técnicos, sino cambiar la educación, organizando procesos que establezcan nuevos valores educativos (Carr y Kemmis, 1988).

A partir de esta perspectiva, el educador debe desarrollar una serie de estrategias docentes emancipadoras, reflexivas y criticas, con las cuales analizar las condiciones sobre las que actúa; esto, desde un trabajo colaborativo, como alternativa a expertos, editoriales y cualquier otra aportación de los tecnócratas que se limitan a reproducir conocimiento sin hacer cuestionamientos críticos.

Los objetivos y los contenidos deben atender una visión integral del sujeto, ayudarle a alcanzar una mayor autonomía para construir su propio aprendizaje; de manera que la comunicación con los escolares sea una de las claves que posibilite el proceso educativo para la construcción social del currículo. Igualmente, la evaluación tendrá un carácter crítico y emancipador, posibilitando a los jugadores instrumentos y procesos de autoevaluación que les ayuden a participar en forma măs activa y más responsable en su propia valoración personal (Tinning, 1992).

A partir de esta tradición, la actividad docente se enfrenta a una estructura ecológica diversa, representada por procesos complejos que se desarrollan en el espacio educativo, desde una visión contextual no generalizable. Ante los problemas de la enseñanza que cambian continuamente, el docente debe desconfiar de una solución definitiva e inmediata y buscar estrategias de acción que le ayuden a actuar en las distintas situaciones.

\section{¿Cuál es el perfil de los educadores vinculados al deporte escolar?}

Revisadas las diferentes perspectivas sobre las cuales vienen formándose los educadores en general y los educadores deportivos en particular, hay que tener en cuenta que un equipo deportivo suele ser reflejo de su 
entrenador; es decir, éste proyecta su personalidad y su forma de hacer al equipo que dirige.

En este apartado se recoge el perfil de los educadores deportivos a partir de las tradiciones educativas, justificado al considerar que los educadores deportivos mantienen y reproducen un tipo de actuación docente similar al resto de enseñantes en sus respectivas labores en el ámbito escolar.

Así, mientras que la tradición empírico-analítica determina los perfiles de los entrenadores autoritarios y tradicionales, la tradición simbólica-interpretativa permite desarrollar los perfiles colaborativo y permisivo, y deja, por último, la tradición crítica para vincular los perfiles creativo y crítico.

Para Saura (1996), el perfil de los técnicos deportivos se define por ser un colectivo muy joven, falto de experiencia, sin continuidad, sin una formación específica y con poca dedicación.

A su vez, la formación de los educadores deportivos es muy heterogénea. No existe uniformidad curricular, tampoco una tradición académica y científica que explique el método a seguir en la formación de esos técnicos deportivos.

La formación de los educadores deportivos españoles es heterogénea. Podemos encontrar educadores de diferentes modalidades deportivas con el mismo nivel de titulación pero con un grado de formación diferente, más grave aún, educadores de la misma modalidad deportiva y con el mismo título que han cursado programas formativos muy dispares (Ibáñez y Medina, 1999).

En el estudio de Ortúzar (2004) se concretan más esas características, gracias a diferentes estudios realizados en diversos contextos españoles (Cataluña, País Vasco, Canarias, Castilla y León, etc.). Se trata de un monitor de género masculino, con una edad de 20 a 29 años, sin ninguna titulación ni formación deportiva.

Por último, Petrus (1997) nos dice que resulta ilógico que una actividad con tanta trascendencia educativa, como el deporte escolar, esté bajo la responsabilidad de educadores con una formación deficiente o nula.

Después de revisar las características del perfil de los educadores deportivos, a partir de Tutko y Richards, (1984), Ibáñez (1996), Fraile (1996), Moreno y Villar (2004), pasaremos a concretar y definir diferentes perfiles de educadores:

\section{El entrenador autoritario}

Se caracteriza por ser una persona enérgica, exigente, que demanda constantemente a los jugadores obediencia y sometimiento a los objetivos y los resultados pre- vistos. Se encarga de tomar todas las decisiones e impone un código personal de actuación que todos han de aceptar (tabla 1). Este perfil no es el más adecuado para las etapas iniciales, cuando a nuestros escolares debemos educarlos hacia un modelo más participativo y democrático. Eso no evita que haya momentos en que el entrenador deba hacer uso de su situación de poder y recriminar a los escolares cuando su comportamiento sea inadecuado.

\section{Carractensticas}

Partidario de la disciplina sin excepciones.

Rígido en el cumplimiento de los programas de entrenamiento.

Amenaza con castigos para motivar a los jugadores en el cumplimiento de las normas.

Persona poco cálida, rechaza las relaciones intimas y mantiene las distancias con los jugadores.

Evita la comunicación con educadores que tienen otros puntos de vista.

Desprecia a los que considera débiles en cumplir sus demandas.

\section{Ventalas}

Los jugadores son disciplinados, y siguen las instrucciones del entrenador.

El equipo está bien organizado en el campo y es físicamente agresivo.

La buena condición física es una de sus armas.

Existe un buen ambiente en el equipo cuando triunfan.

\section{Desvertaris}

Los jugadores sensibles o menos capacitados no soportan las exigencias del técnico como máximo responsable, y actúan en una forma poco segura Temen que sus respuestas no sean del agrado del entrenador.

Hay problemas y conflictos cuando los resultados no acompañan los aprendizajes adquiridos.

Los jugadores se encuentran presionados y tensos, incluso cuando no compiten.

Tablata 1. Perfil del entrenador autoritario.

\section{El entrenador tradicional-conservador}

Sigue un modelo de entrenamiento de eficacia contrastada, a partir de un conocimiento técnico y táctico, sin dejar nada a la improvisación y a modificaciones posteriores. Su metodología es directiva y genera un clima de trabajo en el que todos saben lo que se les va a exigir.

Los medios y los recursos materiales son los específicos y propios de su manifestación deportiva. Es reacio a incorporar nuevos métodos materiales sino se ha comprobado su eficacia. Su trabajo genera un clima de entrenamiento serio, técnico, en el cual todos saben lo que se les va a exigir.

Para este tipo de entrenador, los jugadores son el objeto de su trabajo y los maneja en función de sus intereses. Al ser poco dado a los cambios entre los jugadores veteranos, da pocas oportunidades a los más noveles, busca 
la seguridad y el control del vestuario. Las pocas innovaciones de su práctica profesional parten de patrones ya conocidos, lo cual resulta monótono para los jugadores que llevan más tiempo con él. Es un tipo de entrenador más recomendable en las categorías superiores que en las etapas iniciales, por la falta de ese espíritu innovador, que exige al jugador comportamientos físicos y técnicos que, en ocasiones, no es capaz de realizar.

Podría decirse, en resumen, que busca la seguridad y el control del vestuario a través de los jugadores que le dan más garantías de éxito, y deja escasas posibilidades a los más jóvenes.

\section{El entrenador colaborativo}

Está a favor de un modelo más participativo. Parte de una filosofía por la cual el entrenador y los jugadores se reparten las responsabilidades que han de asumir, para lo cual llegan a acuerdos en las decisiones a través de una postura de diálogo y abierta a la opinión de todos los jugadores. Este tipo de entrenador siempre está preocupado por el bienestar de sus deportistas e inspira respeto sin necesidad de adoptar una postura autoritaria, pues le gusta ser definido como una persona cálida, sociable y que disfruta de su relación con los jugadores (tabla 2).

Gracias a ese proceso democrático obtendremos una buena cohesión en el grupo, jugadores más responsables y autónomos, que actúan a partir de unos principios de carácter personal y no por la imposición externa de un adulto. Por todo ello, este entrenador puede ser uno de los más válidos para fomentar unos valores educativos positivos entre los escolares que se inician en la práctica deportiva, ya que les permite ganar confianza en su aprendizaje.

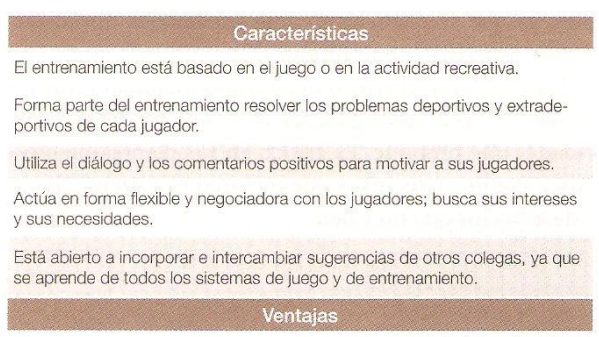

Con esa actitud de apoyo los jugadores rinden por encima de sus posibilidades.

El equipo está relajado y disfruta de la competición.

Existe una buena cohesión del grupo.

Los jugadores "problema" se manejan con menos conflictos.

\section{REFLEXIONES ORI GINALES}

Continuación

\section{Desventeiles}

gunos jugadores veteranos y la propia directiva juzgan al ontronodor cono débil (lo acusan de que no tiene mano derecha).

El entrenador tiene dificultad para manejar a los jugadores poco motivados.

Es inadecuado para jugadores inseguros que necesitan un entrenador autoritario, que le piden soluciones ante su falta de capacidad para toma decisiones en el campo.

Tabla 2. Perfil del entrenador colaborativo

\section{El entrenador permisivo}

Delega en los jugadores la toma de decisiones (tabla 3). Le interesa ganar, pero si pierde lo considera como parte del juego. Este perfil encaja en las etapas iniciales, pues a la vez que se deja a los jugadores tomar la iniciativa, permite observar y detectar la capacidad de los buenos jugadores. No debe confundirse a los educadores permisivos con aquellos despreocupados por su tarea educativa.

El entrenador permisivo deja a los jugadores que tomen la iniciativa, actitud que no siempre es fácil de desarrollar. La mayoría de los técnicos deportivos no deja fácilmente la iniciativa a sus escolares, toma siempre las decisiones por considerar que su labor es la de transmitir su conocimiento.

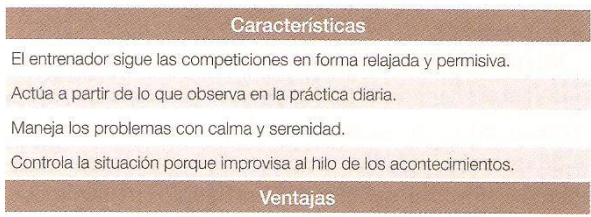

Ejerce poca presión sobre los jugadores y actúa como una persona fria.

No hace que los jugadores entrenen duro, les deja cierta autonomía durante el entrenamiento.

Consigue que los jugadores sean receptivos a sus instrucciones.

Da la impresión de estar poco interesado en su labor.

Los jugadores no están preparados para actuar con autonomía. No es váido para las etapas de rendimiento.

Tabla 3. Perfil del entrenador permisivo.

\section{El entrenador creativo}

Con su estilo de enseñanza innovador trata de potenciar entre sus jugadores técnicas y estrategias poco habituales. En el entrenamiento le gusta experimentar, mantiene lo positivo y diferente. Esa variabilidad de los entrenamientos genera un clima agradable, creativo, incluso divertido, entre los jugadores, ya que se encuentran ante situaciones novedosas y diferentes que evitan 
la rutina y la monotonía. Es un entrenador adecuado a las categorías inferiores, en las cuales la necesidad de ganar debe ser inferior. Sin embargo, ayuda al mejoramiento de la técnica individual, al favorecer el desarrollo creativo del jugador dejándole tomar la iniciativa en su proceso formativo.

\section{El entrenador inconformista-crítico}

Aplica su visión crítica al entrenamiento, a partir de analizar, reflexionar y cuestionar su práctica. Su estilo de entrenamiento está caracterizado por la búsqueda de la perfección, por la corrección del último detalle, por la búsqueda de posibles errores, en un clima de trabajo muy autoexigente ante el entrenamiento y la competición.

Este modelo parte de la premisa de que el enriquecimiento teórico-práctico del entrenador surge del conocimiento tácito que activa y elabora desde su práctica; así, a la vez que experimenta, aprende. La reflexión implica poder sumergirse en forma consciente en el mundo de su experiencia personal y entrar en contacto con sus valores, sus intereses sociales, sus relaciones afectivas, etcétera.

Se trata de una persona que busca su perfeccionamiento en forma continua, apoyándose en la investigación de su práctica, del trabajo en equipo, y en la autoevaluación de su actuación.

Mientras que en la teoría resulta relativamente sencillo definir cada uno de los perfiles presentados, en la práctica no lo es, ya que los educadores suelen valerse de cada uno de ellos en diferentes momentos. También hay educadores fieles a sus principios pedagógicos, comprometidos con unos valores democráticos en su práctica y en una relación digna con los jugadores y que mantienen durante su vida un perfil definitivo.

\section{Conclusiones}

Es preciso establecer una relación pedagógica entre el educador y el jugador a través de estrategias que les ayuden a tomar decisiones, a conseguir una mayor igualdad de oportunidades y a resolver los conflictos en forma de-

\section{Referencias}

Carr, W. y Kemmis, S. (1988). Teoría crítica de la enseñanza. La investigación-acción. En: La formación del profesorado. Barcelona: Ediciones Martínez Roca.

Cook, T. D. y Reichardt, Ch. S. (1986). Métodos cualitativos y cuantitativos en investigación educativa. Madrid: Morata. mocrática. Esto exige consultar a los jugadores y tomar decisiones de manera consensuada, utilizar el debate y la deliberación respetando las posturas de los participantes. También debe permitirse que se cuestionen sus relaciones de poder (como educador), ayudar a que los jugadores aprendan a pensar por sí mismos y a no depender de una autoridad externa que piense por ellos.

Una vez revisados, podemos apreciar que los diferentes estilos de entrenadores y educadores deportivos se caracterizan por atender diferentes perfiles. El autoritario impone sus criterios en forma directa, señala en cada momento lo que debe hacerse y no considera los posibles aportes de los escolares. Incluso, si es preciso, hace uso del castigo cuando los participantes no responden a sus propuestas. Desde esa misma perspectiva, el tradicional se define porque prefiere dirigir y planificar personalmente todo el proceso de entrenamiento desde métodos de eficacia reconocida, y es perfeccionista en la búsqueda de los métodos.

Hacia una dimensión de cooperación, el colaborativo se distingue porque coordina con sus ayudantes la planificación, la aplicación del método de trabajo y la selección de los medios. Le gusta rodearse de ayudantes innovadores pero que, a su vez, sean críticos con el trabajo que realiza.

Al permisivo le gusta enseñar dejando que los jugadores tomen la iniciativa; actitud que es difícil de mantener para la mayoría de los técnicos deportivos, a quienes les cuesta dejar la iniciativa a sus escolares, ya que consideran que su labor está vinculada con la toma de decisiones.

El creativo busca poder experimentar y poner en práctica tareas que generen entre los escolares la creación de gestos técnicos diferentes. Esto favorece que se origine entre los jugadores un clima agradable e incluso divertido, ya que se encuentran ante situaciones novedosas donde no existe la monotonía.

Para finalizar, el crítico tiene como finalidad crear un clima en el cual los jugadores se involucren en las actividades que propone. Es crítico en sus planteamientos, busca medios y recursos que mejoren el entrenamiento y desecha los que no valen.
Elliott, J. (1990). La investigación-acción en educación. Madrid: Morata. Fraile Aranda, A. (2005). Metodología de la enseñanza y entrenamiento deportivo aplicado al fútbol. Madrid: Gymnos. (Coord.). (2004). El deporte escolar en el siglo XXI: análisis y debate desde una perspectiva europea. Barcelona: Graó. 


\section{REFLEXIONES ORIGINALES}

et al. (2001). Actividad física jugada: una propuesta educativa para el deporte escolar. Alcoy: Marfil.

1996). Reflexiones sobre la presencia del deporte en la escue a Educación Física, 46

Giroux, H. A. (1990). Los profesores como intelectuales. Hacia una pedagogía crítica del aprendizaje. Barcelona: Paidós.

Guba, E. (1985). Criterios de credibilidad en la investigación naturalista. En: Gimeno, S. y Pérez Gómez. (1985). La enseñanza: su teoría y su práctica. Madrid: Akal.

Habermas, J. (1987). Teoría y praxis. Madrid: Tecnos.

Ibáñez, S. J. (1996). Análisis del proceso de formación del entrenador español de baloncesto. Tesis doctoral. Universidad de Granada (sin publicar).

Ibáñez, S. J. y Medina, J. (1999). Relaciones del entrenador deportivo y la formación del profesor de Educación Física. Apunts. Educación Física y Deportes, 56.

Kirk, D. (1990). Educación física y curriculum. Valencia: Universidad de Valencia.
Moreno, M. y Del Villar, F. (2004). El entrenador deportivo. Barcelona: Inde.

Ortúzar Oyanguren, I. (2004). Fl deporte escolar y el profesorado de Educación Física. Tesis doctoral. Leioa. Vizcaya.

Pérez Gómez, A. (1988). Pensamiento práctico del profesor: implicaciones en la formación del profesorado. En: A. Vila. Perspectivas y problemas de la función docente. Madrid: Narcea.

Petrus, A. (1997). El deporte escolar hoy. Valores y conflictos. Aula de innovación educativa, 68.

Saura, J. (1996). El entrenador en el deporte escolar. Lleida: Institut D'Estudis Ilerdencs.

Stenhouse, L. (1987). La investigación como base de la enseñanza. Madrid: Morata.

Tinning, R. (1992). Educación física: la escuela y sus profesores. Valencia: Universidad de Valencia.

Tutko, T. A. y Richards, J. W. (1984). Psicología del entrenamiento deportivo. Madrid: Pila Teleña. 